\title{
Resonancia magnética nuclear en el postoperatorio de discectomía lumbar en pacientes asintomáticos y con síndrome de cirugía lumbar fallida
}

\author{
Ressonância nuclear magnética no pós-operatório da discectomia \\ lombar em pacientes assintomáticos e com síndrome de cirurgia \\ lombar falida
}

\author{
Nuclear magnetic resonance in postoperative lumbar discectomy in \\ asymptomatic patients and with failed back surgery syndrome
}

\author{
Armando Alpizar-Aguirre' \\ José Antonio García Rentería ${ }^{2}$
}

\section{RESUMEN}

Objetivo: analizar los cambios posquirúrgicos en pacientes sometidos a discectomía lumbar, tanto en pacientes cuya evolución ha sido satisfactoria, como en aquellos con "Síndrome de Cirugía Lumbar Fallida", enfatizando el justo valor que la resonancia magnética nuclear tiene. Métodos: el universo de trabajo está constituido por dos grupos: Grupo I, cinco pacientes con evolución satisfactoria, asintomáticos; y Grupo II con diez pacientes con resultados considerados no satisfactorios y/o Síndrome Fallido Lumbar. Se analizaron los resultados obtenidos en la imagen de resonancia magnética nuclear, emitiendo un diagnóstico con evaluación externa sin conocimiento del estado clínico de los pacientes. También se realizó la correlación con el estado clínico en ambos los grupos. Resultados: las manifestaciones clínicas preoperatorias tuvieron una distribución muy similar. En ninguno de los dos grupos se reportaron complica-

\section{RESUMO}

Objetivo: analisar as mudanças póscirúrgicas nos pacientes submetidos à discectomia lombar em pacientes com evolução satisfatória, assim como naqueles com "Síndrome de Cirurgia Falida", dando ênfase ao justo valor que a ressonância nuclear magnética tem. Métodos: o universo do trabalho esta constituido por dois grupos, Grupo I com cinco pacientes com evolução satisfatória, assintomáticos. O Grupo II possui dez pacientes com resultados considerados não-satisfatórios elou "Síndrome Falida Lombar". Analisaram-se os resultados obtidos na imagem da ressonância nuclear magnética, emitindo um diagnóstico com avaliação externa sem conhecimento do estado clínico dos pacientes, e realizou-se uma correlação com o estado clínico de ambos os grupos. Resultados: as manifestações clínicas pré-operatórias tiveram uma distribuição muito similar. Em nenhum dos grupos se reportaram complicações transope-

\begin{abstract}
Objective: to analyze the postoperative changes in patients undergoing lumbar discectomy, both in patients whose evolution has been satisfactory, as in those with "Failed lumbar Surgery Syndrome", emphasizing the fair value that the nuclear magnetic resonance has. Methods: the work environment consists of two groups: Group I, five patients with satisfactory evolution and asymptomatic. Group II is composed of ten patients with unsatisfactory results and/or Failed Lumbar Syndrome. The results obtained in the nuclear magnetic resonance image were analyzed, emitting a diagnosis with external evaluation without knowledge of the clinical status of patients, and a correlation with clinical status in both groups was performed. Results: the clinical preoperative manifestations had a very similar distribution. Transoperatory complications were not reported in any of the two groups. In all the groups, the histopathology study was carried out and it was
\end{abstract}

\footnotetext{
Departamento de Neurocirurgía del Hospital de Especialidades del Centro Médico "La Raza" - IMSS - Mexico - D.F.

'Adscrito al Servicio de Cirugía de Columna del Instituto Nacional de Rehabilitación (INR) de la Secretaria de Salud (SS), México.

${ }^{2}$ Neurocirujano, Consultante del Servicio de Neurocirugía del Centro Médico "La Raza" - IMSS - Mexico - D.F. 
ciones transoperatórias. En todos se estudió por histopatología y se reportó disco degenerado o hialinizado. En el Grupo I, no se encontró imagen de alguna anomalía a pesar de su estado clínico. En todos los pacientes se encontraron datos de fibrosis posquirúrgica; en cuatro casos, disco residual con compresión radicular en los cinco pacientes. En el Grupo II, sólo en ocho pacientes, la resonancia magnética nuclear demostró algún hallazgo anormal. Los hallazgos anormales fueron: fibrosis posquirúrgica en cinco casos $(50 \%)$, disco residual en tres casos $(30 \%)$ y datos de compresión radicular en seis de los casos $(60 \%)$. Conclusión: no hay congruencia entre los hallazgos de resonancia magnética nuclear y la clínica, en pacientes postoperados de discectomía lumbar con evolución satisfactoria, hasta el momento, asintomáticos. La resonancia magnética nuclear no es confiable en estos pacientes.

DESCRIPTORES: Imagen por resonancia magnética; Síndrome de fracaso de la cirugía espinal lumbar; Columna vertebral/cirugía ratórias. Em todos os grupos, foi feito o estudo histopatológico e foi informado o disco degenerado ou hialinizado. No Grupo I, não foi encontrada imagem de alguma anomalia apesar de seu estado clínico. Em todos os pacientes foram encontrados dados de fibroses pós-cirúrgica, em quatro casos, disco residual, com compressão radicular nos cinco pacientes. No Grupo II, só foi possivel encontrar em oito pacientes, e a ressonância nuclear magnética demonstrou algum achado anormal, os quais foram fibroses pós-cirúrgica, em cinco casos (50\%); disco residual, em três casos (30\%); e dados de compressão radicular, em seis casos (60\%). Conclusão: não há congruência entre os achados da ressonância nuclear magnética e a clínica em pacientes pós-operatórios da discectomia lombar com evolução satisfatória, até o momento, assintomáticos. A ressonância nuclear magnética não é confiável nestes pacientes.

DESCRITORES: Imagem por ressonância magnética; Síndrome pós-laminectomia; Coluna vertebral/cirurgia reported the disk degenerated or hyalinization. In Group I, it was not found any abnormality, despite its clinical state. In all the patients, postoperative fibrosis data was found in four cases, and residual disk root was compressed in five patients. In Group II, only in eight patients, the nuclear magnetic resonance showed abnormal findings. The abnormal findings were: fibrosis postoperative in five cases $(50 \%)$, residual disk in three cases $(30 \%)$, and data root compression in six cases (60\%). Conclusion: there is no congruence between the nuclear magnetic resonance and clinic findings, in postoperative patients, of lumbar disectomy with a satisfactory development, who, so far, are asymptomatic. The nuclear magnetic resonance is not reliable in these patients.

KEYWORDS: Magnetic resonance imaging; Failed back surgery syndrome; Spine/surgery

\section{INTRODUCCIÓN}

La evaluación imagenológica de la columna demanda no solamente la capacidad de visualizar las estructuras óseas, es necesario también poder evaluar la médula espinal, las raíces nerviosas y el saco tecal. La imagenología de la columna ha cambiado desde 1997 con la introducción de nuevos métodos de estudio, como la ultrasonografía, la tomografía computada (TC), la resonancia magnética nuclear $(\mathrm{RMN})$, y la tomografía computarizada por emisión fotónica única (SPECT). Los datos obtenidos de éstas técnicas diagnósticas de corte seccional permiten obtener una localización tridimensional de los diferentes procesos patológicos que afectan la columna y proveen información adicional para caracterizar la lesión, obteniendo un grado más alto de sensibilidad y especificidad que otras modalidades diagnósticas ${ }^{1,2}$.

Aunque la RMN no provee la alta definición ósea como la TC, la apropiada combinación de secuencias de pulsos otorga ventaja en la caracterización de los diferentes tejidos para discriminar el tejido óseo del resto de los tejidos ${ }^{3,4}$.
Recientemente, se ha incrementado el uso de la RMN en diferentes procesos patológicos, sobre todo en aquellos en donde la caracterización tisular es esencial, como en la evaluación del paciente post operado de columna lumbar, en donde el cirujano se enfrenta a un reto diagnóstico ${ }^{5,6}$. Por lo tanto, es importante conocer los cambios estructurales antes y después del evento quirúrgico en aquellos pacientes que así lo ameritan ${ }^{7,8}$. Dado que varía de un tejido a otro, la RMN puede proporcionar una imagen detallada de las estructuras del cuerpo, incluyendo el cráneo y la columna ${ }^{1}$. El contraste tisular ofrece una imagen detallada de los diferentes tejidos, sin la necesidad de algún procedimiento intervensionista. Puesto que es posible la realización de imágenes en múltiples planos, relativamente grandes áreas de la columna pueden ser evaluadas inicialmente, en un plano sagital y adicionalmente, en un plano axial en los niveles de mayor interés. Siendo todo esto posible, sin la exposición a radiación, en contraste con la mielografía, TC ó placas simples, aunque con el inconveniente de no ofrecer imágenes en carga o en movimiento?. 
La capacidad de la RMN para manipular el contraste entre los diferentes tejidos blandos y óseos ofrece importantes ventajas sobre las diferentes modalidades de imagen mencionadas anteriormente, sin el riesgo que implica la introducción de contraste exógeno en el espacio subaracnoideo $^{10}$.

Aunque la RMN demuestra claramente los defectos extradurales, puede ser en ocasiones difícil en imágenes en $\mathrm{T} 1$ diferenciar la baja intensidad de un disco protruido de un osteofito que no es muy grande como para contener grasa del tejido esponjoso. La correlación de los diferentes ecos, así como las imágenes sagitales y oblicuas a través del foramen neural, sirven para dilucidar dicha situación, según esté el defecto extradural contiguo al cuerpo vertebral o al disco intervertebral, especialmente en la columna cervical.

La administración intravenosa de material paramagnético (Gadolinium - DTPA), con una dosis de $0.1 \mathrm{mmol} /$ $\mathrm{kg}$ de peso corporal, es utilizada para la evaluación de algunas enfermedades intramedulares. Mientras en las secuencias T1 y T2 se localiza satisfactoriamente la lesión, el Gadolinium ayuda mediante su reforzamiento a distinguir el tumor del edema perilesional, quistes intratumorales y necrosis, o sirinx adyacente, además de proveer cierto patrón vascular. El reforzamiento es útil también para diferenciar neoplasias de procesos inflamatorios ${ }^{9}$.

Sin embargo, no todas son ventajas en la RMN, el costo de esta modalidad e imagen es relativamente alto en comparación a otras alternativas diagnósticas. Otra desventaja de la RMN es que no en todos los pacientes es posible realizarla, aquellos con marcapasos cardiacos, clips aneurismáticos ferromagnéticos, otros implantes también ferromagnéticos (por ejemplo, implantes cocleares, etc.), y con cuerpos extraños intraoculares deben ser excluidos. Un pequeño porcentaje de los pacientes sometidos a estudios de RMN puede presentar cuadros de claustrofobia que, sin embargo, pueden ser solucionados con una leve sedación.

En la actualidad, la RMN se considera como la modalidad de imagen de elección para el estudio del postoperatorio de la columna lumbar, su capacidad de imagen multiplanar, su capacidad de resolución tisular, así como su caracterización, son sus mayores ventajas. La introducción de material paramagnético para el reforzamiento selectivo de algunas estructuras de la columna postoperada, junto con la variedad de imágenes sin contraste, ha incrementado su sensibilidad y especificidad. La evolución de los nuevos métodos de la RMN es y seguirá siendo un proceso continuo.

La discectomía lumbar es un procedimiento realizado en conjunción con una laminectomía. Los cambios postdiscectomía son generalmente evidentes en las diferentes imágenes. Una imagen en $\mathrm{T} 1$ en el postoperatorio inmediato permite observar tejido blando anormal anterior al saco tecal en el sitio original de la herniación discal, tejido que se observa de intensidad intermedia. Este tejido se confunde finamente con la porción posterior del disco en el sitio de la discectomía. En las imágenes en T2, este tejido presenta un incremento importante en su intensidad, frecuentemente este puede ser tan grande como la hernia discal original y producir un efecto de masa en el saco dural anterior. La cantidad de éste tejido extradural se reduce gradualmente en los meses siguientes a la cirugía. La altura del disco también suele disminuir con el paso del tiempo, hasta poder ocasionar una estenosis del receso lateral. Otro hallazgo característico asociado con la discectomía es la presencia de disrupción anular, ésta es vista tanto en los cortes axiales como en los sagitales del T2 en el postoperatorio inmediato ${ }^{11,12}$.

En la RMN, los sitios de laminectomía son fácilmente identificables. Tanto en el plano axial como en el sagital se observa la ausencia de dichas estructuras normales, pérdida de la alta intensidad de la médula ósea laminar en $\mathrm{T} 1$, y pérdida de la baja intensidad del hueso cortical tanto en imágenes en T1 como en T2. El sitio de la laminectomía sufre una serie de cambios con el paso del tiempo, las imágenes obtenidas en el postoperatorio inmediato muestran una masa irregular de media intensidad heterogénea en las imágenes en T1, y de elevada intensidad en el T2. Este tejido blando presumiblemente es resultado del edema postoperatorio, obliterando los planos musculares y grasos de la musculatura paraespinal a lo largo del trayecto quirúrgico. En los meses siguientes, la intensidad media de este tejido se vuelve más homogénea en el T1, y pierde parte de su intensidad en las imágenes en T2, producto del origen de la formación de la cicatrización. El saco tecal mantiene o recupera regular contorno redondeado y es frecuentemente desplazado, posteriormente, hacia el sitio de la laminectomía, al tiempo que protruye dentro del tejido del lecho ${ }^{13,14}$.

Dentro de los hallazgos anormales comunes en el postoperatorio de la patología lumbar, tenemos: estenosis espinal lateral, herniación discal residual o recurrente, fibrosis postoperatoria, aracnoiditis, pseudoartrosis, lesión neural durante la cirugía, infección posquirúrgica, calcificación distrófica y osificación, las cuales se asocian en mayor parte de los casos con una evolución tórpida del paciente $^{15-18}$.

Las causas del síndrome de cirugía lumbar fallida han sido enumeradas por diferentes autores, las dividiendo en dos grupos:

- No mecánica: fibrosis posquirúrgica, aracnoiditis, lesión neural indirecta, enfermedad sistémica, inestabilidad psicosocial, infección y ganancia secundaria.

- Mecánica: estenosis central o lateral, disco residual, nivel erróneo, cuerpo extraño, hematoma, pseudoartrosis, espondilolistesis, pseudos-artrosis, espondilolistesis, pseudomeningocele y disco a otro nivel $1^{11,12,18-22}$.

Con la pregunta de investigación de ¿Tiene la Imagen de Resonancia Magnética Nuclear, la suficiente confiabilidad, en el estudio del paciente post-operado de discectomía lumbar con evolución tórpida, como para en base 
a dicho estudio emitir un diagnóstico certero y decidir su manejo médico o quirúrgico?

El presente estudio se enfoca a analizar los cambios posquirúrgicos en pacientes sometidos a discectomía lumbar por hernia discal, tanto en pacientes cuya evolución ha sido satisfactoria, como en aquellos con "Síndrome de Cirugía Lumbar Fallida", que representa aún actualmente un problema clínico, desde el punto de vista diagnósti$\mathrm{co}^{10,}, 23$. Se enfatiza el justo valor que tiene la RMN en el estudio de dichos pacientes. El objetivo es analizar las alteraciones estructurales en pacientes postoperados de discectomía lumbar por hernia discal, mediante imagen de resonancia magnética nuclear, tanto en pacientes con evolución satisfactoria como en aquellos con evolución tórpida, con correlación con su estado clínico. También tiene como objetivo determinar el grado de confiabilidad del diagnóstico de imagen de la RMN en el paciente postoperado de discectomía lumbar o por hernia discal, mediante el cotejo de dicho diagnóstico de imagen y su evolución y estado clínico.

Pensando que la imagen de resonancia magnética nuclear, a pesar de ser actualmente el estudio de imagen de elección en el estudio del paciente postoperado de discectomía lumbar con evolución tórpida, y a pesar de sus importantes ventajas con respecto a otras modalidades de imagen, no posee la suficiente confiabilidad, como para en base a un diagnóstico de imagen de dicho estudio, emitir un diagnóstico certero y sobre todo decidir el manejo médico o quirúrgico más adecuado en estos pacientes. La clínica, por tanto, sigue siendo el parámetro más confiable para la elección del manejo médico o quirúrgico en dichos pacientes.

\section{MÉTODOS}

El universo de trabajo está constituido por dos grupos de pacientes quienes fueron sometidos a cirugía de columna lumbar por patología del disco intervertebral lumbar, específicamente por hernia de disco lumbar con radiculopatía y/o ciatalgia secundaria, en el Departamento de Neurocirugía del Hospital de Especialidades del Centro Médico "La Raza", con técnicas quirúrgicas de hemisemilaminectomía para un solo disco o laminectomía para dos o más discos contiguos, con su discectomía correspondiente. Se incluye un total de 15 pacientes formando dos grupos:

- Grupo I- cinco pacientes quienes por su evolución posoperatoria, seguimiento por consulta externa y estado actual se considere con reintegración completa a sus labores habituales; con ausencia de dolor lumbar o radicular; y sin déficit neurológico.

- Grupo II - diez pacientes quienes por su evolución y seguimiento postoperatorio, los resultados son considerados no satisfactorios y presentan Síndrome Fallido Lumbar, o sea, incapacidad laboral. Dolor lumbar y/o radicular; déficit neurológico.

Se realizó una entrevista clínica y exploración física a los pacientes tanto del Grupo I como del Grupo II, posterior a lo cual se les realizó un estudio de Imagen de resonancia magnética nuclear; con equipo General Electric de 0.5
Tesla del Hospital de Especialidades del Centro Médico "La Raza", incluyendo: proyección sagital T1 localizador; proyección axial y sagital en T1 y T2; proyección axial y sagital en T1 con administración intravenosa de Gadolinium DTPA a dosis de $0.1 \mathrm{mmol} / \mathrm{kg}$ de peso corporal.

Se analizaron los resultados obtenidos en la imagen de resonancia magnética nuclear, emitiendo un diagnóstico imagenológico, sin conocimiento por parte de los asesores (Jefe del Departamento de Neurocirugía y Jefe del Departamento de Radiodiagnóstico del HECM "La Raza"), del estado clínico del paciente en el postoperatorio.

Posterior a la interpretación de los estudios de IRMN, se realizó una correlación con el estado clínico de cada uno de los pacientes de ambos grupos.

\section{RESULTADOS}

Del total de pacientes del Grupo I, fueron cuatro mujeres (80\%) y un hombre (20\%), con una relación M:H de cuatro a uno. Con una edad media de 48.6 años, con rango de 40 a 64 años. La duración media de los síntomas preoperatorios fue de 30.8 meses, con un rango de dos meses a diez años. En dos casos hubo afección de tres somitas (40\%); en un caso, de dos (20\%); y en los dos restantes, una (40\%), Los miotomos más afectados fueron: L5 en cuatro pacientes $(80 \%)$, S1 en cuatro pacientes $(80 \%)$ y L4 en dos pacientes $(40 \%)$. Los dermatomas más afectados en el fueron L5 en tres pacientes $(60 \%), \mathrm{S} 1$ en tres pacientes $(60 \%)$ y L4 en un paciente (20\%). En el $100 \%$ de los casos en ambos grupos se presentó lumbalgia, y dolor radicular en cuatro pacientes $(80 \%)$. Los estudios de imagen usados fueron: radiografías simples en los cinco pacientes (100\%), RMN en un paciente $(20 \%)$ y en cuatro pacientes mielotomografía y radiculografía. Los discos más afectados fueron: L4 en tres pacientes $(60 \%)$, y L4 en tres pacientes $(60 \%)$.

Del total de pacientes del Grupo II, fueron seis mujeres $(60 \%)$ y cuatro hombres $(40 \%)$, con una relación M:H de 1.5 a 1 . Con promedio de edad de 40.2 , con un rango de 37 a 62 años; duración de sintomatología preoperatorio de 28.8 meses, y con rango de 3 meses a 8 años. En un caso hubo afección de seis $(10 \%)$, en un caso de cinco $(10 \%)$, en un caso de cuatro (10\%), en un caso de tres $(10 \%)$, en dos casos de dos $(20 \%)$ y en los cuatro casos restantes, un solo (40\%). Los miotomos más afectados fueron L5 en nueve pacientes $(90 \%), \mathrm{S} 1$ en seis pacientes (60\%), L4 en cuatro pacientes (40\%), L3 en dos pacientes $(20 \%)$ y finalmente L2 en un paciente (10\%). Las dermatomas más afectadas fueron: L5 en siete pacientes $(70 \%), \mathrm{S} 1$ en cuatro pacientes $(40 \%)$, L4 en tres pacientes $(30 \%)$ y L3 en dos pacientes $(20 \%)$. Lumbalgia en todos los casos y radiculopatía solo en nueve, con correspondencia a las hematomas afectadas. Los estudios de imagenología utilizados fueron radiografías simples en los 10 casos (100\%); en cuatro casos, RMN (40\%); en dos casos, radiculografía y mielotomografía (20\%); y en un caso $(10 \%)$ solamente tomografía. Los discos más afectados fueron: L4 en ocho pacientes ( $80 \%)$, L5 en cuatro pacientes $(40 \%)$, L3 en dos (20\%), y L2 en un paciente (10\%). 
Las manifestaciones clínicas preoperatorios en ambos los casos fueron: dolor lumbar bajo, dolor radicular, paresia de uno o más miotomos, hipoestesia en uno o más dermatomas, signos de tensión radicular y reflejos de estiramiento muscular disminuidos o ausentes.

De los pacientes del Grupo I, a cuatro pacientes se les realizó hemisemilaminectomía del nivel afectado $(80 \%)$, a uno $(20 \%)$ laminectomía por estar involucrados niveles contiguos, a la totalidad de ellos se les practicó discectomía del disco afectado (100\%). Los pacientes tuvieron una estancia intra-hospitalaria media de 6.6 días con un rango de 3 a 12 días. Todos tuvieron evolución satisfactoria con recuperación ad integrum y con reintegración completa de sus actividades cotidianas.

De los pacientes del Grupo II, se les realizó hemisemilaminectomía a ocho pacientes $(80 \%)$; a dos pacientes, laminectomía (20\%). Los pacientes tuvieron una estancia intra-hospitalaria media de 6.3 días con un rango de 3 a 11 días. Y presentaron paresia de algún miotomo en ocho casos (80\%), hipostesia de algún dermatoma en ocho casos $(80 \%)$, dolor lumbar bajo en ocho casos $(80 \%)$ y dolor radicular en siete casos $(70 \%)$. Los miotomos más afectados fueron: L5 en siete casos (70\%), S1 en tres casos (30\%), L4 en dos casos (20\%), y 13 en un solo caso (10\%). Los dermatomas afectados fueron: L5 en ocho casos (80\%), S1 en tres casos (30\%), y L4 en solo un caso (10\%).

En ninguno de los dos grupos se reportaron complicaciones transoperatórias. En todos los casos se enviaron los discos resecados para comprobación histopatológica, en todos ellos se reportó disco generado o hialinizado.

El tiempo medio de realización de la RMN fue en el Grupo I de 20.6 meses, en el Grupo II de 16.1 meses, con un rango de 4 a 36 meses y de 3 a 55 meses, respectivamente.

Como se mencionó a todos los pacientes, se les realizó un estudio de RMN con el equipo General Electric de 0.5 Tesla, en postoperatorio. Las imágenes obtenidas fueron interpretadas por el Jefe del Departamento de Radiodiagnóstico y el Jefe del Departamento de Neurocirugía del Hospital, sin conocimiento de los casos ni el estado clínico del paciente. Los diagnósticos emitidos fueron los siguientes:

- Grupo I: en ninguno de los casos se encontraron sólo hallazgos considerados como normales, el 100\% de ellos se observó en la imagen alguna anomalía a pesar de su estado clínico. En el total de los pacientes (100\%) se encontraron datos de fibrosis posquirúrgica, en cuatro casos $(80 \%)$, disco residual con compresión radicular en los cinco pacientes (100\%), en ninguno se encontró canal lumbar estrecho.

- Grupo II: sólo en ocho pacientes (80\%), la resonancia magnética demostró algún hallazgo anormal, en los dos restantes $(20 \%)$, sólo se encontraron hallazgos considerados como normales, a pesar de su estado clínico. Los hallazgos anormales fueron: fibrosis posquirúrgica en cinco casos (50\%), disco residual en tres casos (30\%) y datos de compresión radicular en seis de los casos $(60 \%)$.

\section{DISCUSIÓN}

De los grupos estudiados, el mayor número de pacientes en ambos los grupos fueron del sexo femenino, el grupo etario más afectado en el Grupo I fue la quinta década, en el Grupo II la cuarta. La edad media del Grupo I también fue mayor.

El cuadro clínico preoperatorio en ambos los grupos tuvo una duración media muy similar, 30.8 meses en el Grupo I y 28.8 meses, en el Grupo II.

Las manifestaciones clínicas preoperatorias tuvieron una distribución muy similar, siendo las más frecuentes (en el 100\% de los casos), dolor lumbar bajo y afección de algún miotomo.

En el Grupo II, la sintomatología preoperatoria fue más florida, con un número mayor de miotomos y dermatomos afectados, hasta seis miotomos y cuatro dermatomas, a una diferencia del Grupo I en que el mayor número de miotomos y dermatomos afectados fueron tres y tres, respectivamente. Los miotomos afectados más frecuentemente en ambos grupos fueron L5 y S1 con predominio del primero, el cual estaba involucrado en el $80 \%$ de los pacientes del Grupo I y en el $90 \%$ de los pacientes del Grupo II. Los dermatomas más afectados fueron los mismos, L5 y S1 de predominio L5.

Debido a la infraestructura y los recursos del hospital, en el Grupo I el estudio más usado para establecer el diagnóstico preoperatorio fue la mielotomografía en el $80 \%$ de los casos, en el $40 \%$ la RMN y en el $30 \%$, radiculografía solamente. En todos los casos las placas simples fueron de importancia fundamental (100\% de los casos en ambos los grupos).

El procedimiento quirúrgico realizado con mayor frecuencia fue la hemisemilaminectomía en el $80 \%$ de los pacientes en ambos grupos, y sólo en dos casos del Grupo II se realizó foraminotomía por canal lumbar estrecho lateral, y en otros dos laminectomía por estenosis central.

Hubo más incidencia de canal lumbar estrecho en el Grupo II, en el Grupo I no se presentó ningún caso. Los discos afectados en el Grupo I fueron solamente dos, L4 y L5, en el 60\% de los casos en ambos casos, en el Grupo II los discos involucrados fueron cuatro, L2, L3, L4 y L5, con mayor frecuencia L4 en el 80\% de los casos, seguido de L5 en el $40 \%$, después L 3 en el $20 \%$ y finalmente L2 en el $10 \%$.

El cuadro clínico posquirúrgico en los pacientes del Grupo II con síndrome fallido lumbar estuvo constituido por dolor lumbar, dolor radicular, afección de algún miotomo y afección de algún dermatoma con una frecuencia de presentación muy similar (80\%).

Contrasta el hallazgo realizado en la serie de estudios de RMN realizado a estos pacientes de ambos grupos, en los que se observó, en el Grupo I de pacientes asintomáticos, una total incongruencia entre los hallazgos encontrados y la clínica del paciente, observando datos clásicos y muy evidentes de fibrosis posquirúrgica en la totalidad de los casos, disco residual en cuatro de los casos, con compresión radicular franca desde el punto de vista imagenológico en cuatro de ellos. En el Grupo II existió más congruencia en 
los hallazgos de RMN, sólo en dos casos se observaron únicamente cambios posquirúrgicos considerados como normales, así mismo se realizó el diagnóstico de canal lumbar estrecho en el $30 \%$ de los casos, a diferencia del diagnóstico preoperatorio en el que se realizó dicho diagnóstico solamente en el 10\% de los casos. En el Grupo II también el hallazgo imagenológico más frecuentemente realizado fue la fibrosis posquirúrgica, seguido de disco residual y canal lumbar estrecho, con compresión radicular secundaria.

\section{CONCLUSIONES}

No hay congruencia entre los hallazgos de RMN y la clínica, en pacientes postoperados de discectomía lumbar con evolución satisfactoria, hasta el momento, asintomáticos. La RMN no es confiable en estos pacientes.
Hay congruencia entre los hallazgos de RMN y la clínica en aquellos pacientes postoperados de discectomía lumbar, con evolución tórpida característica del síndrome fallido lumbar. La RMN es más confiable en pacientes con síndrome fallido lumbar que en asintomáticos.

Debe efectuarse especial seguimiento a largo plazo, a los pacientes postoperados de discectomía lumbar, especialmente asintomáticos, ya potencialmente puede convertirse en sintomáticos.

La clínica sigue siendo la que determina la pauta del manejo médico o quirúrgico del paciente postoperado de columna lumbar. La relación médico y paciente es fundamental.

Las imágenes más útiles en el estudio del paciente postoperado de discectomía lumbar son: sagital T1 con y sin Gadolinium, y axial T1 con Gadolin.

\section{REFERENCIAS}

1. Bates D, Ruggieri P. Imaging modalities for evaluation of the spine. Radiol Clin North Am. 1991;29(4):675-90.

2. Czervionke LF. Lumbar intervertebral disc disease. Neuroimaging Clin $\mathrm{N}$ Am. 1993;3(3):465-85.

3. Modic MT, Steinberg PM, Ross JS, Masaryk TJ, Carter JR. Degenerative disk disease: assessment of changes in vertebral body marrow with MR imaging. Radiology. 1988;166(1 Pt 1):193-9.

4. Pech P, Haughton VM. Lumbar intervertebral disk: correlative MR and anatomic study. Radiology. 1985;156(3):699-701.

5. Hueftle MG, Modic MT, Ross JS, Masaryk TJ, Carter JR, Wilber RG, et al. Lumbar spine: postoperative MR imaging with Gd-DTPA. Radiology. 1988;167(3):817-24.

6. Jinkins JR. Magnetic resonance imaging of benign nerve root enhancement in the unoperated and postoperative lumbosacral spine. Neuroimaging Clin N Am. 1993;3(3):485-97.

7. Wiesel SW, Tsourmas N, Feffer HL, Citrin CM, Patronas N. A study of computer-assisted tomography. I. The incidence of positive CAT scans in an asymptomatic group of patients. Spine (Phila Pa 1976). 1984;9(6):549-51.

8. Yu S, Haughton VM, Rosenbaum AE. Magnetic resonance imaging and anatomy of the spine. Radiol Clin North Am. 1991;29(4):691-710.

9. Atlas SW. Rationale and clinical indications for contrast agents in MR imaging of the brain and spine. J Comput Assist Tomogr. 1993;17:Suppl 1:S1-7.
10.Bundschuh CV. Imaging of the postoperative lumbar spine. Neuroimaging Clin N Am. 1993;3(3):499-515.

11. Ross JS, Masaryk TJ, Modic MT, Bohlman H, Delamater R, Wilber G. Lumbar spine: postoperative assessment with surface-coil MR imaging. Radiology. 1987;164(3):851-60.

12.Djukic S, Genant HK, Helms CA, Holt RG. Magnetic resonance imaging of the postoperative lumbar spine. Radiol Clin North Am. 1990;28(2):341-60.

13. Boden SD, Davis DO, Dina TS, Parker CP, O’Malley S, Sunner JL, et al. Contrast-enhanced MR imaging performed after successful lumbar disk surgery: prospective study. Radiology. 1992;182(1):59-64.

14.Ross JS. Magnetic resonance assessment of the postoperative spine. Degenerative disc disease. Radiol Clin North Am. 1991;29(4):793-808.

15. Hochhauser L, Kieffer SA, Cacayorin ED, Petro GR, Teller WF. Recurrent postdiskectomy low back pain: MR-surgical correlation. AJR Am J Roentgenol. 1988;151(4):755-60.

16. Youmans JR. Neurological surgery: a comprehensive reference guide to the diagnosis and management of neurosurgical problems. 3rd ed. Philadelphia: Saunders; 1990.

17.Long DM. Failed back surgery syndrome. Neurosurg Clin N Am. 1991;2(4):899-919.

18. Boden SD, Wiesel SW. The multiply operated low back patient. In: Rothman RH, Simeone FA, editors. The spine. 3rd ed. Philadelphia: Saunders; 1992.
19. Teplick JG, Haskin ME. Intravenous contrast-enhanced CT of the postoperative lumbar spine: improved identification of recurrent disk herniation, scar, arachnoiditis, and diskitis. AJR Am J Roentgenol. 1984;143(4):845-55.

20.Sotiropoulos S, Chafetz NI, Lang P, Winkler M, Morris JM, Weinstein $\mathrm{PR}$, et al. Differentiation between postoperative scar and recurrent disk herniation: prospective comparison of MR, CT, and contrast-enhanced CT. AJNR Am J Neuroradiol. 1989;10(3):639-43.

21. Weinbergm ST. Disc disease, spondilosis and related disorders. In: Pomeranz SJ, editor. Craniospinal magnetic resonance imaging. Philadelphia: Saunders; 1989. p. 541-65.

22.Ross JS, Masaryk TJ, Modic MT, Delamater R, Bohlman H, Wilbur $\mathrm{G}$, et al. MR imaging of lumbar arachnoiditis. AJR Am J Roentgenol. 1987;149(5):1025-32.

23.Jinkins JR, Osborn AG, Garrett D Jr, Hunt S, Story JL. Spinal nerve enhancement with Gd-DTPA: MR correlation with the postoperative lumbosacral spine. AJNR Am J Neuroradiol. 1993;14(2):383-94.

\section{Autor para correspondencia:}

Armando Alpizar Aguirre

Camino a Santa Teresa - 1055-684

Col. Héroes de Padierna

Delegación Magdalena Contreras

CP: 10700

México, D. F.

E-mail: aalpizar@inr.gob.nx 